\title{
A Novel Method for Despeckling of Ultrasound Images Using Cellular Automata-Based Despeckling Filter
}

\author{
Ankur Bhardwaj, ASET, Amity University, India \\ Sanmukh Kaur, Amity University, India \\ Anand Prakash Shukla, KIET Group of Institutions, India \\ Manoj Kumar Shukla, Amity University, India
}

\begin{abstract}
Ultrasound images have an inherent property termed as speckle noise that is the outcome of interference between incident and reflected ultrasound waves which reduce image resolution and contrast and could lead to improper diagnosis of any disease. In different approaches for reducing the speckle noise, there exists a class of filters that convert multiplicative noise into additive noise by using algorithmic functions. The current study proposes a cellular automata-based despeckling filter (CABDF) that implements a local spatial filtering framework for the restoration of the noisy image. In the proposed CABDF filter, a dual transition function has been designed which emphasizes the calculation of nearby weighted separation whose loads originate from the CABDF filtered image, including spatial separation, extend inconsistency, and statistical dispersion. The proposed filter found efficient both in terms of filtering and restoration of the original structure of the ultrasound images.
\end{abstract}

\section{KEYWORDS}

Cellular Automata, Despeckling Filters, MSE, MSSIM, PSNR, SNR, Speckle Noise, SRMSE

\section{INTRODUCTION}

Ultrasound (US) Imaging is a portable, accurate, and pervasive healthcare technology used in medical diagnostics. The ultrasound images are obtained by processing echo signals reflected by body tissues. Its characteristics like low cost, harmlessness, instant results make it more adaptable. It provides realtime pictures of human organs like kidney, gallbladder, stomach, liver, and many more. Ultrasound imaging mode has proven to be very useful in the assessment of the disease of patients, as it is a safe, painless, inexpensive, and speedy process when compared to the other available method (Zhou et. al,2019).

Ultrasound imaging technology helps to treat the patient in a more specific manner which finally directly or indirectly impacts the health of the patient. Unlike other tomographic techniques, ultrasound imaging offers interactive visualization of the underlying anatomy in real-time. Speckle noise, which is multiplicative in nature reduces fine details and limits the contrast resolution in an image This limits the detection of small, low contrast lesions in the body that restrict the proper diagnostics of body tissues. The assessment of US pictures is exclusively relying upon the expertise of the specialist and speckle noise works as a barrier in the analysis. Further potential issues and some existing solutions are provided for denoising. (Singh et. al, 2020) 


\section{BACKGROUND}

In the earlier decades, different algorithms have been introduced for speckle denoising including local spatial filters described by Guo et.al (2009), Gabriel et.al (2014) explained anisotropic diffusion (AD) filters, Guo et.al (2011) discussed non-local means (NLM) filters. Jose and Mario (2010) explained the total variation (TV) methods for filtering. Charles Alban et.al (2017) enhanced the previously existing homomorphic approaches. Homomorphic filtering method transforms multiplicative noise into additive one by use of the logarithmic function. Lee Filter (Jongsen Lee,1980) used the least square approach from the computed values of the window. Kuan (Darwin T Kuan et. al,1985) described a filter known as the Kuan filter which employed wavelet-based channels that have been broadly utilized as a result of its versatile behavior.

Achim et. al., (2001) utilized the overwhelmingly followed alpha-stable distribution to show sub-band disintegrations and planned a Bayesian processor to evacuate speckle. Two newly additive gaussian filters named block-matching and three-dimensional filtering (BM3D) (Davoc et. al,2007) and dual-domain image denoising (DDID) (Knaus and Zwicker,2013) work in a homomorphic manner for despeckling. Khare introduced a speckle noise contraction method based on Daubechies wavelet transformation that recognizes the solid edges and segments of complex wavelet coefficients (Khare et al.,2010).

Zhang introduced a wavelet shrinkage fast bilateral filter(WSFBF) algorithm (Zhang et al., 2016) that used the concept of the fast bilateral filter and the Bayesian shrinkage algorithm to process the low pass coefficients. Another approach analyzing the wavelet shrinkage and guided channel ( $\mathrm{He}, \mathrm{K}$ et. al,2016) could be found in (Zhang et. al,2016). Inferable from the multiresolution and directional attributes of wavelet change, multiscale techniques successfully save texture information, however, they will, in general, introduce ringing artifacts. Popovici planned a nearby change capacity with the end goal that the present cell's state is a component of the past conditions of the neighboring cells (Popvici et al, 2002). Be that as it may, these strategies are not ideal since these are sceptic to the nearness of neighborhood 25 highlights like edges and thus yield dull images particularly for high noise density. A Uniform CA rules-based filter (Selvapeter and Hordik,2009) decides that a 2-D CA with an elementary standard for reducing impulse noise. Shukla portrays a strategy for the determination of the best cellular automata rule set from a huge search space based on the sequential floating forward search technique (Shukla et. al,2014). Mangalam and Vekatesh(2017) extemporized a recombination algorithm to apply the idea of cellular automata in the grayscale images to expel salt and pepper noise from the image. Bhardwaj et.al (2019) proposed a filter that is based on cellular automata and applied it to the Moore neighborhood in the image. Here in CA-based rules the boundary pixels are eliminated and not considered for denoising. Multiscale strategies right off the bat embrace wavelet change to decay the first picture or its logarithmic structure into sub-band coefficients, which are then handled by methods for some all-around planned plan that can wipe out the element of speckle. Me. $\mathrm{K}$. Hu et.al (2019) used phase asymmetry (PAS) to maintain the equilibrium between total variation and anisotropic diffusion despeckling filter. Mukerjee et.al (2019) iteratively reduced the size of the image fidelity for noise reduction. Rotman et. al(2019) proposed an algorithm to reduced speckle noise along with image compression. Latif et. al (2020) proposed a CNN model-based algorithm for the breast ultrasound dataset and achieved significant accuracy. (Salazar et. al,2020) discussed the despeckling methods which have an impact on legal metrology for healthcare services mainly determined on human safety. Alex and Chandy (2020) changed the edges and different features in the evaluation of inpainting in speckled noisy images. The concept of non-local filter used here shows the statistical metric based on the ratio between two patches on breast tumor datasets (Ambrosanio et. al, 2020).

Lastly, the filtered image is obtained by inverse wavelet transformation. (Wenchao Cui et. al,2020) depicted a guided trilateral channel that re-established a noisy image with a well-fitting statistical distribution model. Figure1 shows the classification of filters used for denoising. 


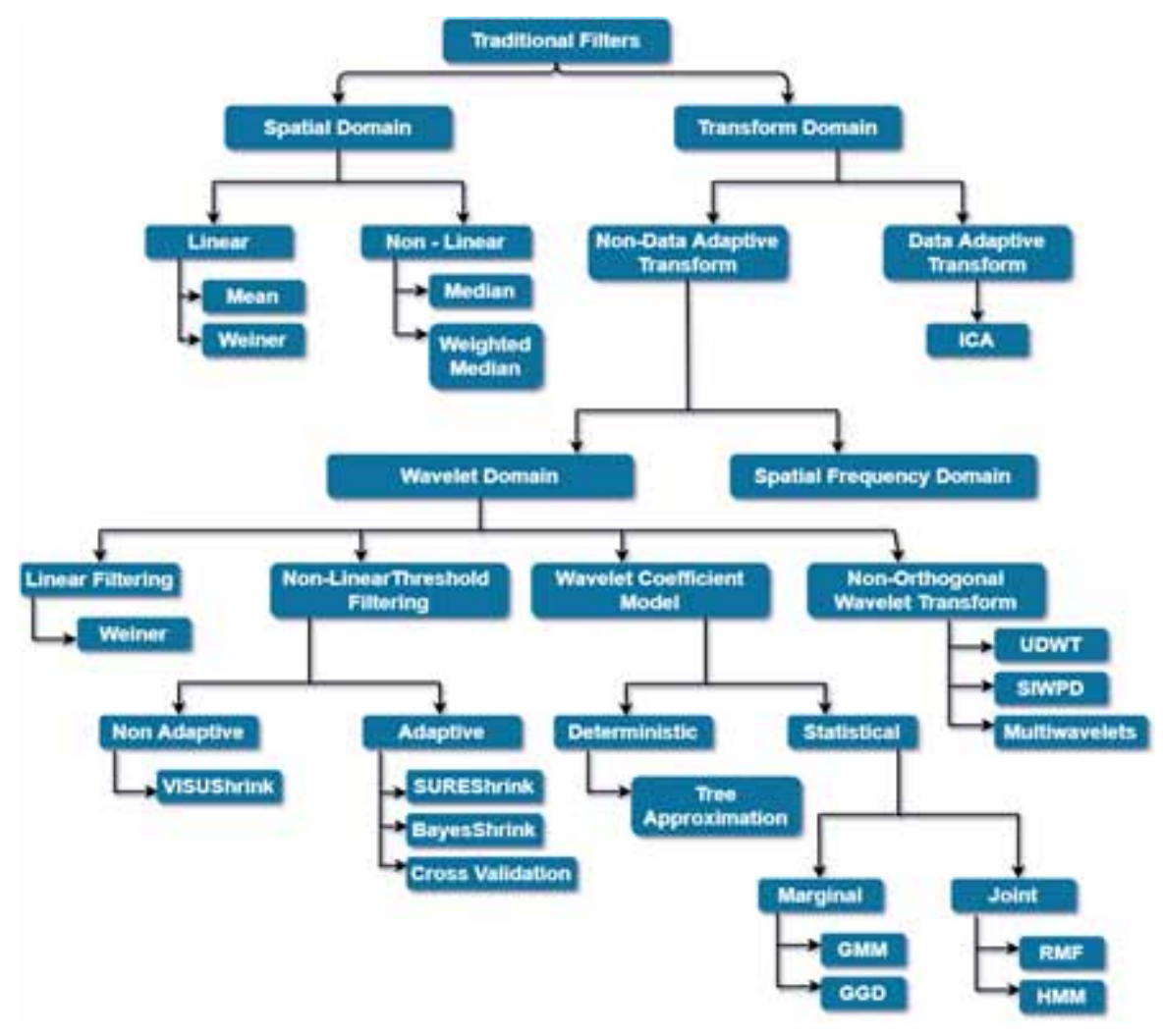

A novel cellular automaton-based despeckling filter (CABDF) is proposed which depends on the neighborhood's most extreme probability estimation from the probabilities of the residuals between the CABDF filtered image and the speckle noise corrupted image. In the current paper impact of different existing despeckling filters on the US, images are being examined. Filters executed in a system and the outcome is based on different image-based parameters as square root mean square error(SRMSE), Signal-to-noise ratio(SNR), peak signal to noise ratio(PSNR), mean square error(MSE), structural similarity(SSIM). The given filter retains the structure as well as reduce the noise from the image affected by speckle noise. The whole filter is used the cellular automata's concept of tuples in image processing. This transition function is the most dynamic part of the whole calculation. We applied a cellular automata-based transition function for despeckling and it gives better results than other despeckling filters. In this paper we have shown our associated work in systematic and well-organized manner (Tiwari et.al,2016). The proposed method is performing the filtering in the digital form of ultrasound images and it can be easily uploaded and processed in mobiles for despeckling.

\section{CELLULAR AUTOMATA MODEL}

Cellular Automaton(CA) model for the methodology on images is portrayed by a 2-D matrix-like architecture with an individual cell having a specified state that is investigated a great deal of limitedly various decision at every discrete time step. Additionally, this state revised in time as per a particularly depicted plan of rules dealing with the movement of CA which may consider a past filled up with all past states of the cell and its neighbors. This 'rulebook' is usually time-invariant and taken independently from the cell being alluded to in the question (wolfram S.,1984). 


\section{Structure of Cellular Automata}

A 2-D deterministic CA can be comprehended and characterized as an arranged three tuples:

$\mathrm{A}=(\mathrm{S}: \mathrm{N} ; \delta)$

where $\mathrm{S}$ is a non-empty State set. A standard way to define neighborhood ${\mathrm{N} \epsilon \mathrm{Z}^{2}}^{2}$ is to use a ball defined by a metric $\mathrm{N}(\mathrm{a} 0, \mathrm{~b} 0)$ (Popovici A. and Popovici D.,2002). Two types of variety of neighborhood are:

- Von Neumann Neighbourhood: Here a kind of the diamond shape approach used with its neighbor which is depicted in figure 2(a)and is used to define a cell set with specified neighboring cell range $\mathrm{N}(\mathrm{a} 0, \mathrm{~b} 0)$ which influences the square grid of 2D CA. Von Neumann for range $\mathrm{r}$ could be represented as:

$\mathrm{N}(\mathrm{a} 0, \mathrm{~b} 0)=(\mathrm{a} 0, \mathrm{~b} 0):|\mathrm{a}-\mathrm{a} 0|+|\mathrm{b}-\mathrm{b} 0| \leq \mathrm{r}$

- Moore Neighbourhood: The square shape neighbourhood as described in figure 2(b) is used to traverse over the pixel in this type, even the set of specified neighbouring cells $\mathrm{N}(\mathrm{a} 0, \mathrm{~b} 0)$ are used for definition. For range $r$ it can be represented as:

$\mathrm{N}(\mathrm{a} 0, \mathrm{~b} 0)=(\mathrm{a}, \mathrm{b}):|\mathrm{a}-\mathrm{a} 0| \leq \mathrm{r}, \mathrm{lb}-\mathrm{b} 0 \mid \leq \mathrm{r}$

The Cellular automaton was initially depicted by (Ulam,1972) as a discrete dynamical system. A Discrete system can be termed as the parameters of space and that of time. Here the lattice (cells) contains the fixed number of states that are organized in some particular kind of pattern. The dimensions of the grid can be fixed with any number. The state of a particular lattice at any point of time depends only on itself and on the neighboring cells at one step ahead. The state of the cell is constructed by some kind of transformation rule which can be a kind of mathematical function and changes accordingly and hence updated simultaneously. Where ' $\delta$ ' a is a transition function that conflicts with the present state of the provided cell and its neighbors and provides the next state of the assigned cell as indicated by the deterministic rule. The kernel patterns, positioning of state, and the ruleset $(\delta)$ besides the initial states thoroughly describe a particular CA.

Figure 2. (a)Von Neuman (b) Moore Neighbourhood

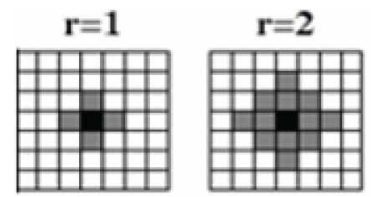

(a)

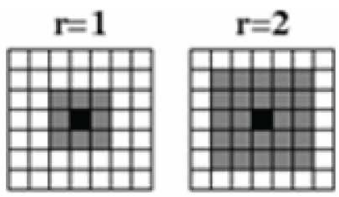

(b) 


\section{FILTERS FOR SPECKLE NOISE REDUCTION}

\section{Lee Filter}

Proposed by (J.S. Lee, 1980) founded on diversity over an area, and the variation is made delicate only if it is small or constant over an area. We can calculate the power of the signal of the average pixel cell in the filter window using the least square method from the estimated value of the described cell. Lee's filter works fine with the retention of the edges. The Lee filter can be built like:

$$
I_{i, j}=\bar{I}+W^{*}\left(C_{p i}-\bar{I}\right)
$$

where $\mathrm{I}$ is the pixel values post-filtering, $\bar{I}$ filter the neighbourhood, the $\mathrm{C}_{\mathrm{pi}}$ center pixel, $\mathrm{W}$ is the average value of the intensity of the filter neighbour weighted function. Lee filter didn't work well to remove speckle noise at edges.

\section{Kuan filter}

The Kuan Filter (Kuan et. al.,1985) is viewed as a superior filter to the Lee filter. The filter uses fundamentally the same way as the real signal to deal with an impedance for the neighbourhood center pixel, and it is expected that the speckle noise pursued a negative exponential propagation as well as produces a near average, with the probability function which includes the center pixel and standard deviation noise (Rukmani and Singh,2008). Its weighted function 'W' is described as:

$$
W=\frac{\left(1-\frac{C_{u}}{C_{i}}\right)}{1+C_{u}}
$$

where $\mathrm{Cu}$ is the calculated noise variance coefficient and $\mathrm{Ci}$ is the variation coefficient of the image.

\section{Lee Diffusion Filter}

Typically, Lee filter works on the $7 \times 7$ sliding window. In Lee Diffusion Filter, we treat the internal window filter window as $(x-1, y),(x, y-1),(x+1, y),(x+1, y)$. Lee filter can be depicted as discrete isotropic dispersion.

\section{SRAD filter}

The Speckle Anisotropic Diffusion Filter performs de- speckling and maintains image edges without changing its valuable information. The SRAD acts as a mean filter, and preserves the edges and attributes better than the regular methods such as frost, lee, and kuan filters (Sasikala D. and Madheswaran M.,2014).

\section{Anistropic Diffusion filter}


Anisotropic Diffusion (PMAD) (Perona and Malik,1990) filter uses the approach of nonlinear partial differential equations and is characterized as:

$$
\begin{aligned}
& \frac{\partial I}{\partial t}=\operatorname{divg}[\operatorname{cof}(|\nabla I|) \cdot \nabla I] \\
& \mathrm{I}(\mathrm{t}=0)=\mathrm{I} 0
\end{aligned}
$$

Here $\nabla$ is the gradient operator, the divg divergence,|operator denotes the magnitude, cof (x) is the diffusion coefficient, and I0 the initial image. The two diffusion coefficients are:

$$
\operatorname{cof}(x)=\left(\frac{1}{1+\left(\frac{x}{k}\right) 2}\right)
$$

and:

$$
\operatorname{cof}(x)=\exp \left[\left(-\frac{x}{k}\right)^{2}\right]
$$

Frost filter (Yu and Acton,2002) is the convolutional linear despeckling filter. Frost filter is based on the coefficient of variation which is the proportion of local standard deviation to the local mean of the noisy image. Inside $\mathrm{N} \times \mathrm{N}$ neighbourhood the middle pixel value is change by weighted summation of the values of neighbors of the pixel. A weighting factor is depreciated while moved forward from the center pixel of concern and raised along with variance.

\section{Wavelet Filter}

Donoho initiated the wavelet based noise removal employing thresholding of the Discrete Wavelet Transform (DWT) coefficients (Donoho and Johnstone,1995). Wavelets have been utilized for the noise reduction of images for over 20 years. Wavelet change is a multiresolution impression of signal and image in two dependent areas, which decay the signal and image into multiscale goals. The limitation of the wavelet premise works in both time and fourier domain prompts multiresolution analysis and compelling filter designs for specified applications.

\section{Linear Scale}

In the linear scale filtering method we consider a window of $\mathrm{M} \times \mathrm{N}$ and then calculate the average of all pixels whose difference in the intensity level $\mathrm{I}_{\mathrm{x}, \mathrm{y}}$ and applied to center pixel, is below or equal to a predefined threshold $T$. We assign this value to the intensity level $I_{x, y}$ along with $T=\alpha$ Imax, with Imax, is the highest intensity level of the image and $\alpha=[0,1]$. Linear Scale filter scales the pixel intensities by obtaining the minimum $\mathrm{I}_{x, y}$, and maximum $\mathrm{I}_{\mathrm{x}, \mathrm{y}}$ intensity level values in rotating kernel and then assign the value to the center pixel with: 


$$
F_{x, y}=\frac{I_{\max }+I_{\min }}{2}
$$

\section{IMAGE QUALITY MATRICES}

\section{Mean Square Error (MSE)}

The mean square error is the average of the assigned input image and the square output amplitude of the improved output image and is described as:

$$
M S E=\frac{1}{M N} \sum_{i=1}^{M} \sum_{j=1}^{N}\left(g_{i, j}-f_{i, j}\right)^{2}
$$

It is the most widely used quality measurement parameter. For the $\mathrm{M} \times \mathrm{N}$ window, it calculates the difference in quality between the given input and the processed image (Chen et.al, 2003). The MSE is directly related to the deterioration of the image. A zero MSE has shown a good restored image quality. Therefore, the higher value of MSE describes the lower quality of the image.

\section{Square Root Mean Square Error (SRMSE)}

Square-root-mean-square error (SRMSE) (Prakash et.al, 2011) is the most commonly used method of variance between predicted values for the model or measure and the values seen. SRMSE works to consolidate the magnitude of errors in various predictions. We have to take the square root of MSE to calculate the amount of SRMSE. Mathematically it is defined as:

$$
S R M S E=\sqrt{\frac{1}{M N}} \sum_{i=1}^{M} \sum_{j=1}^{N}\left(g_{i, j}-f_{i, j}\right)^{2}
$$

3. Peak Signal-to-Noise Ratio (PSNR) and Signal-to- noise ratio (SNR)

Peak signal-to-noise ratio (Hore \& Ziou,2010), is the statistical quality measurement parameter is the proportion between the maximum strength and the destructive noise effect of a signal that affects the reliability of its representation. The SNR test is calculated as the pixel difference between the original insert and the restored images. The high value of the PSNR leads to high quality images. Mathematically PSNR is described as:

$$
\text { PSNR }=-10^{*} \log 10 \frac{M S E}{g_{\max }^{2}}
$$


The structural similarity index (Hore \& Ziou,2010) between two images, is given by:

$$
\operatorname{SSIM}(x, y) \frac{\left(2 \mu_{x} \mu_{y}+c_{1}\right)\left(2 \sigma_{x y}+c_{2}\right)}{\left(\mu_{x}^{2}+\mu_{y}^{2}+c_{1}\right)\left(\sigma_{x}^{2}+\sigma_{y}^{2}+c_{2}\right)}
$$

- $\mu_{x}$ : average of $\mathrm{x}$

- $\mu_{y}:$ average of $\mathrm{y}$

- $\sigma_{x}^{2}$ : variance of $\mathrm{x}$

- $\sigma_{y}^{2}$ : variance of $\mathrm{y}$

- $\sigma_{x y}$ : covariance $\mathrm{x}$ and $\mathrm{y}$

\section{PROPOSED CELLULAR AUTOMATA BASED DESPECKLE FILTER (CABDF)}

The essential thought basic proposed filtering is to do in the range of an image what conventional filters do in its domain. Two pixels can be near to each other, that is, involve close by spatial area and neighbours, or they can be like each other, that is, have close by values, potentially in a perceptually meaningful fashion. The spatial distribution of image intensities assumes no role in range filtering. Consolidating intensities from the whole image, in any case, makes little sense, since the dissemination of image values far away from ' $X$ ' should not influence the last value at ' $X$ '. Moreover, one can show that range filtering without area separating scarcely changes the colormap of a picture, and is along these lines of little use. The proper arrangement is to merge the domain and range filtering. A coloured or grey digital image is characterized as an $\mathrm{M} \times \mathrm{N}$ with $\mathrm{M}$ rows and $\mathrm{N}$ columns. The same provision could be accepted for the cross arrangement of 2-D CA where each cell like a pixel of an image and realistic conditions of cells are the binary, grey, or chromatic estimations of comparing pixel. At first, the worth is characterized as the pixel esteems characterized by input image. The state of the cell is depending upon the state of the neighbourhood cells. For denoising dark scale ultrasound pictures between [0 255] and Moore neighbourhood is inspected. The proposed filter that appeared in equation (1) has been illustrated. It shows the level of compiled samples in the Moore neighbourhood. In CABDF every pixel is replaced by a proposed transition rule $\delta$. The proposed filter works on a dual transition function named as $\delta 1$ and $\delta 2$ respectively. The transition function $\delta 1$ is the output that is obtained by applying median filtering on the neighbourhood of the target pixel. Thereafter a transition function $\delta 2$ is the cellular automata based filter and then applied on the transition function $\delta 1$. The output is the outcome of convolution between transition function $\delta 1$ and $\delta 2$. This perspective is significant because it makes it simple to secure instinct about its conduct, to adjust it to application-explicit necessities, and to actualize it. It relies just upon two parameters that show the size and contrast of the components to conserve. The main uniqueness of the derived filter is that it retains the edge components during denoising because it did not perform averaging across edges. Conceptually CA processed in parallel. This graphical processing unit functions admirably as it deals with the idea of the multiprocessor. To show the result simulation central processing unit computers have been used for simulation of transition function at each pixel simultaneously. The transition function $\delta$ can be defined as:

$\mathrm{X} 1=$ distance between pixels $\forall P \in N$

$\mathrm{X} 2$ = difference between intensity values $\forall P \in N$ 
$\delta_{1}(P)=\operatorname{med} P_{r}(i, j) ; r=1,2,3, \ldots n$

$\delta_{2}(P)=\sum_{i=1}^{n}\left(X 1_{i} \times X 2_{i}\right)$

$\delta=\delta 1 \times \delta 2$

To normalize the value of $\delta$ it could be divided by a parameter $\mathrm{W}$ then:

$\delta^{\prime}=\frac{1}{W} \delta$

where $\mathrm{P}$ is the pixels in the neighbourhood, $\mathrm{N}$ is the number of pixels in the image and $\mathrm{W}$ is the normalization parameter. The Proposed filter reduced noise as well as retains the edges.

\section{PROPOSED ALGORITHM}

The temporary input image is considered for simulation and the processed pixels are stored in this temporary image. The yield kept in the temporary image is additionally repeated to a unique image at every cycle. The steps of the proposed algorithm as below.

\section{Description of Algorithm}

For each pixel location in speckle noise effected image $(i, j)$ :

Step 1: Consider a Moore neighbourhood with radius ' $r$ ' $(r=1)$ around pixel (i,j) to be denoise.

Step 2: In cellular pattern, the Moore neighbourhood considered eight neighboring pixels around

$(i, j)$ are: (i-r,j-r),(i,j-r),(i+r,j-r),(i-r,j),(i+r,j),(i-r,j+r),(i,j+r), (i+r,j+r).

Step 3: Calculate the transition function $\delta 1=$ median pixel $P(i, j)$ for radius $r$.

Step 4: Calculate the distance $X 1$ between the neighboring pixels in the neighbourhood.

Step 5: Calculate the difference in grey level amplitude values $X 2$ between neighbourhood pixels.

Step 6: Compute transition function:

$\delta 2=\mathrm{X} 1-\mathrm{X} 2$

Step 7: Compute the dual transition function $\delta^{\circ}=\delta 1(\delta 2)$ for 1 to $\mathrm{N}=\mathrm{X} 1 \times \mathrm{X} 2$.

Step 8: Normalized the value by using a normalization factor ' $W$ ' and compute:

$\delta^{\prime}=\frac{1}{W} \delta$

Step 9: Apply the transition function $\delta^{\prime}$ and computer the Cellular Automata based filtered image.

Step 10: Replace the value of Pixel $(i, j)$ with transition function value $\delta^{`}$.

Algorithm 1: Cellular Automata based Despeckling Filter

Result: Filtered Image B

$A=$ input image of size $m \times n$; 


$$
\begin{aligned}
& \text { for } i \leftarrow 2 \text { to } m-1 \text { do } \\
& \text { for } j \leftarrow 2 \text { to } n-1 \text { do }
\end{aligned}
$$

compute $\mathrm{X} 1$ = distance $\mathrm{X} 1$ between the neighboring pixels;

compute $\mathrm{X} 2$ = difference in grey level amplitude values;

$$
\begin{gathered}
\delta 2=x 1-x 2 ; \\
\delta=\delta 1(\delta 2) ; \\
\delta^{\prime}=\frac{1}{W} \delta
\end{gathered}
$$

Apply the transition function $\delta$ 'to $A(i, j)$;

$$
B(i, j)=\delta \cdot A(i, j) \text {; }
$$

end

\section{RESULTS AND DISCUSSION}

The proposed filter is applied on brachial plexus images and simulated on MATLAB 2018a. The quality of images measured on the basis of peak signal to noise ratio, structured similarity index, signal to noise ratio, mean square error, mean square root error on different intensities of speckle noise varies from 0.01 to 0.1 . Table 1 , Table 2 , Table 3 , and Table 4 shows the comparative study of CABDF with eight other well-known despeckling filters.

We considered the density of speckle noise of variance 0.01 and 0.05 respectively and it is introduced to the US test images image 1 and image2. Table 1,2,3 and Table 4 shows the comparison of different filters based on different well-known quality measurement parameters of image1[fig 3, fig 4] and image2[fig 5, fig 6]. Here the data set of Ultrasound Nerve Segmentation has been considered which analyses nerve structures in US images of the neck called brachial plexus in ultrasound imaging from Kaggle (2016) which consists of total of 5500 images. The samples of 100 random clinical images (Kaggle,2016) are considered for testing. Table 1,2,3,4 shows the values of selected image

Table 1. Comparison between different filters on image1 at noise level 0.01

\begin{tabular}{|c|c|c|c|c|c|c|c|c|c|}
\hline Feature/Filters & $\begin{array}{c}\text { Anistropic } \\
\text { Diffusion }\end{array}$ & Kuan & Lee & $\begin{array}{c}\text { Lee } \\
\text { Diffusion }\end{array}$ & $\begin{array}{c}\text { Frost } \\
\text { Diffusion }\end{array}$ & SRAD & Wavelet & $\begin{array}{c}\text { Linear } \\
\text { Scale }\end{array}$ & $\begin{array}{c}\text { Proposed } \\
\text { CABDF }\end{array}$ \\
\hline MSE & 70.61 & 78.31 & 41.31 & 40.40 & 42.68 & 113.06 & 70.22 & 80.13 & 36.52 \\
\hline SNR & 11.15 & 10.254 & 15.81 & 16.00 & 15.52 & 7.06 & 11.20 & 10.05 & 65.07 \\
\hline SRMSE & 8.40 & 8.85 & 6.43 & 6.36 & 6.53 & 10.63 & 8.38 & 8.95 & 6.04 \\
\hline PSNR & 29.68 & 29.23 & 32.00 & 32.10 & 31.68 & 27.63 & 29.70 & 29.13 & 32.53 \\
\hline SSIM & 0.967 & 0.975 & 0.976 & 0.976 & 0.976 & 0.966 & 0.938 & 0.970 & 0.999 \\
\hline
\end{tabular}

Table 2. Comparison between different filters on image1 at noise level 0.05

\begin{tabular}{|c|c|c|c|c|c|c|c|c|c|}
\hline Feature/Filters & $\begin{array}{c}\text { Anistropic } \\
\text { Diffusion }\end{array}$ & Kuan & Lee & $\begin{array}{c}\text { Lee } \\
\text { Diffusion }\end{array}$ & $\begin{array}{c}\text { Frost } \\
\text { Diffusion }\end{array}$ & SRAD & Wavelet & $\begin{array}{c}\text { Linear } \\
\text { Scale }\end{array}$ & $\begin{array}{c}\text { Proposed } \\
\text { CABDF }\end{array}$ \\
\hline MSE & 1530.25 & 144.13 & 108.24 & 111.25 & 43.13 & 549.04 & 139.62 & 80.26 & 52.04 \\
\hline SNR & -15.56 & 4.95 & 7.44 & 7.20 & 15.43 & -6.66 & 5.23 & 10.04 & 62.001 \\
\hline SRMSE & 39.11 & 12.01 & 10.40 & 10.55 & 6.56 & 23.43 & 11.82 & 8.95 & 7.214 \\
\hline PSNR & 16.32 & 26.58 & 27.82 & 27.70 & 31.14 & 20.77 & 26.72 & 29.12 & 31.01 \\
\hline SSIM & 0.642 & 0.933 & 0.934 & 0.929 & 0.976 & 0.861 & 0.896 & 0.969 & 0.999 \\
\hline
\end{tabular}


Table 3. Comparison between different filters on image2 at noise level 0.01

\begin{tabular}{|c|c|c|c|c|c|c|c|c|c|}
\hline Feature/Filters & $\begin{array}{c}\text { Anistropic } \\
\text { Diffusion }\end{array}$ & Kuan & Lee & $\begin{array}{c}\text { Lee } \\
\text { Diffusion }\end{array}$ & $\begin{array}{c}\text { Frost } \\
\text { Diffusion }\end{array}$ & SRAD & Wavelet & $\begin{array}{c}\text { Linear } \\
\text { Scale }\end{array}$ & $\begin{array}{c}\text { Proposed } \\
\text { CABDF }\end{array}$ \\
\hline MSE & 43.65 & 44.68 & 23.96 & 23.54 & 42.83 & 80.57 & 28.99 & 35.82 & 22.96 \\
\hline SNR & 15.33 & 15.12 & 20.54 & 20.69 & 15.49 & 10.01 & 18.88 & 17.04 & 69.10 \\
\hline SRMSE & 6.606 & 6.684 & 4.895 & 4.85 & 6.54 & 8.97 & 5.38 & 5.98 & 4.79 \\
\hline PSNR & 31.77 & 31.66 & 34.37 & 34.45 & 31.85 & 29.10 & 33.54 & 32.62 & 34.55 \\
\hline SSIM & 0.965 & 0.981 & 0.978 & 0.978 & 0.976 & 0.964 & 0.966 & 0.980 & 0.999 \\
\hline
\end{tabular}

Table 4. Comparison between different filters on image2 at noise level 0.05

\begin{tabular}{|c|c|c|c|c|c|c|c|c|c|}
\hline Feature/Filters & $\begin{array}{c}\text { Anistropic } \\
\text { Diffusion }\end{array}$ & Kuan & Lee & $\begin{array}{c}\text { Lee } \\
\text { Diffusion }\end{array}$ & $\begin{array}{c}\text { Frost } \\
\text { Diffusion }\end{array}$ & SRAD & Wavelet & $\begin{array}{c}\text { Linear } \\
\text { Scale }\end{array}$ & $\begin{array}{c}\text { Proposed } \\
\text { CABDF }\end{array}$ \\
\hline MSE & 1081.36 & 84.15 & 61.26 & 62.63 & 61.64 & 385.83 & 71.16 & 74.43 & 38.38 \\
\hline SNR & -12.54 & 9.63 & 12.38 & 12.19 & 12.33 & -3.59 & 11.08 & 10.69 & 64.64 \\
\hline SRMSE & 32.88 & 9.17 & 7.83 & 7.91 & 7.85 & 19.64 & 8.43 & 8.627 & 6.195 \\
\hline PSNR & 17.83 & 28.91 & 30.29 & 30.20 & 30.27 & 22.30 & 29.64 & 29.45 & 32.32 \\
\hline SSIM & 0.663 & 0.940 & 0.939 & 0.939 & 0.940 & 0.862 & 0.928 & 0.952 & 0.999 \\
\hline
\end{tabular}

quality matrices. Table 1,3 shows that Cellular Automata based filter for noise density 0.01 gives the best results in terms of mean square error (Chen et.al, 2003) for low noise level for table 1 and table 3. For noise density level 0.05 From table 2, the MSE value in SRAD filter takes slightly margin than the proposed filter. But in table 4 the MSE value is good enough then all rest of the filters. The SNR value is considered low if its value is less than 24. It is considered good when lies between 25 to 36 . It could be considered as excellent if its value is greater than 36 . Here from the tables shown it is clear that the value of SNR is always considered excellent as it is always greater than 36 . The value of SNR is far away in all other existing filters in comparison to CABDF. The Square root of mean square error is a kind of error that is always better when it is closed to zero. Here except table 2 CABDF works very well then all other discussed filters. Peak signal to noise ratio(PSNR) is considered to be better in all cases in the proposed CABDF. The value of PSNR is always higher in all conditions and noise levels. During despeckling by using any of the filters there is always a problem related to the structure of the image. In previously used filters kuan, lee, lee diffusion, frost diffusion, and linear scale provides a better result related to the structure of the image. But our proposed filter revolutionizes the SSIM value in the image. The CABDF retains the structure of the image and preserves edges for different images at a variant noise level. By applying any of the filters it is the huge problem that the fine details of the filtered image are removed which could lead to wrong observation. Higher the PSNR, SSIM value and lower the MSE, SRMSE value, gives better result.

The graph shown in Figures 7,8,9,10 shows the statistical analysis of different filters along with CABDF, which is based on Tables 1,2,3, and 4. The proposed CABDF results better in all such parameters than the subsequent filters. Hence it is clear from the above findings that transition rules used in the proposed Cellular Automata Based Filter reduces the speckle noise as well as preserves the edges of the filtered image which means it preserves the structure of the ultrasound images. Preserving structure is one of the important factors in diagnosis. 


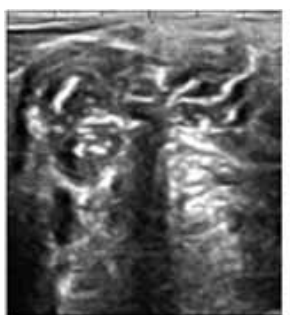

(a) Original

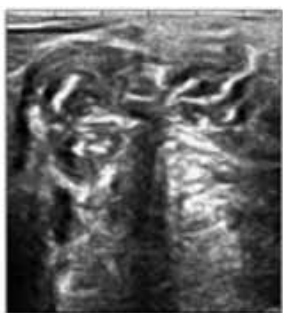

(b) Noise 0.01

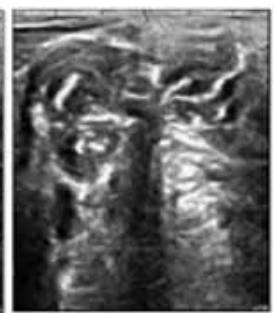

(c) Anistropic Diffusion

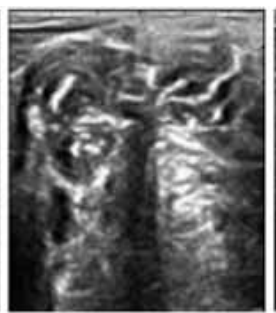

(d) Kuan

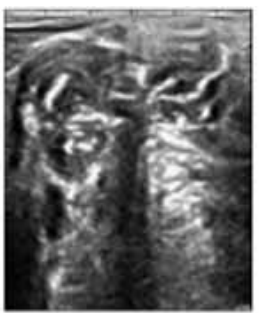

(e) Lee

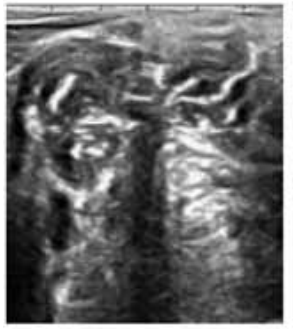

(f) Lee Diffusion



(g) Frost Diffusion

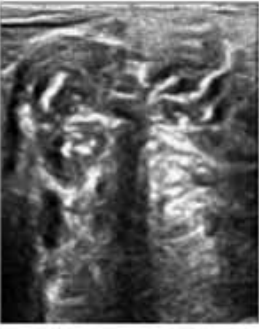

(h) SRAD

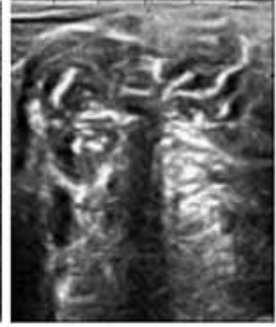

(i) Wavelet

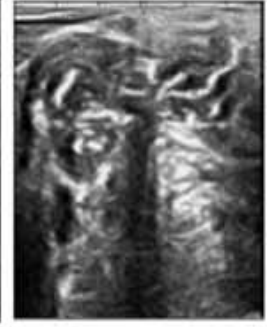

(j) Linear Scale

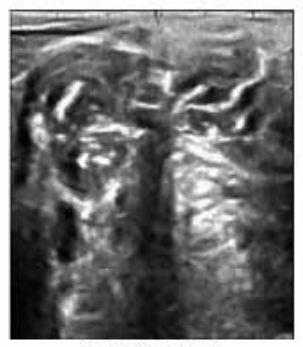

(k) $\mathrm{CABDF}$

\section{CONCLUSION}

Despeckling of the medical ultrasound images is one of the important and challenging tasks in the field of digital healthcare. Overall diagnosis depends on the quality of the ultrasound image obtained. In this paper, Cellular Automata based despeckling filter(CABDF) has been proposed for despeckling of the medical ultrasound images. In this, a novel transition function of cellular automata has been defined after analyzing a large number of existing filters. The proposed filter is implemented in MATLAB and tested in more than 100 randomly selected ultrasound images of brachial plexus form ultrasound nerves segmentation. It has been observed that this filter works efficiently and produces a better quality result. it is compared both qualitatively and quantitatively. To perform the qualitative analysis, the filtered images are compared visually and it is found that identification and diagnosis are easier in these filtered images due to better quality filtering. To perform the qualitative analysis, the results are quantified on the basis of image quality parameters. The result is also compared with some standard and popular filters and it has been found that the proposed filter performs better in various aspects. One of the most important findings from the obtained results and comparison with the standard filters is that the proposed filter is on the top for structure preservation of the images. The structure preservation is one of the important factors for proper and accurate diagnosis from the ultrasound image and the proposed filter perform it very efficiently. 


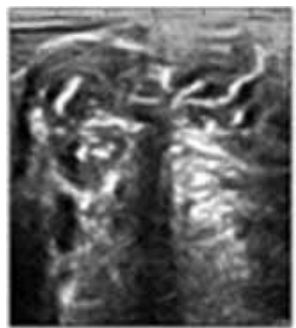

(a) Original

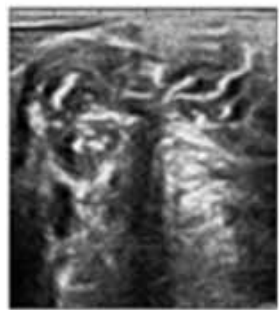

(f) Lee Diffusion

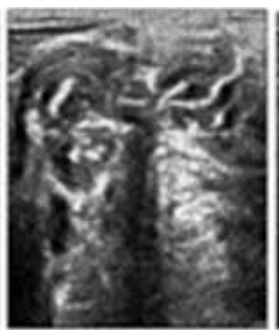

(b) Noise 0.05

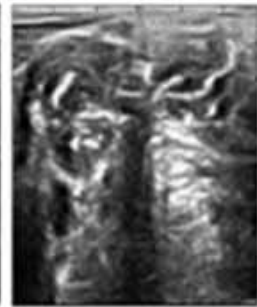

(g) Frost Diffusion

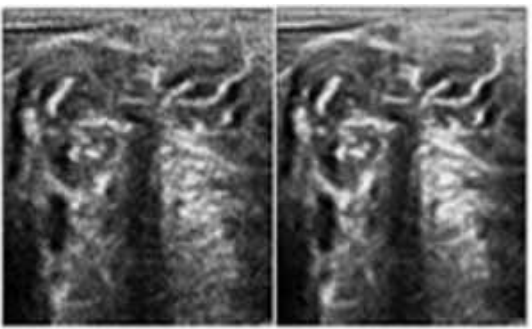

(d) Kuan

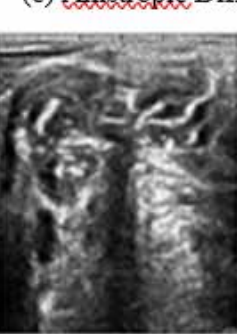

(h) SRAD

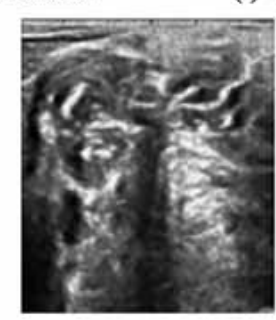

(k) $\mathrm{CABDF}$

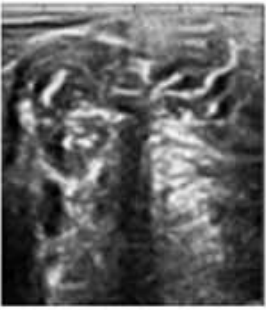

(i) Wavelet

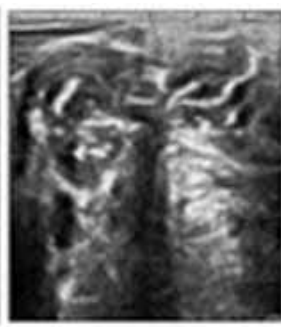

(e) Lee

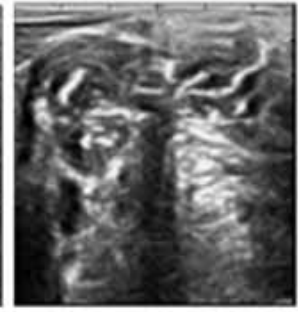

(j) Linear Scale

(k) $\mathrm{ABDF}$

In the future, one may try to design a new type of transition function for the cellular automata which are used for filtering the medical ultrasound images. The design of the transition function and filter may be on the basis of the various criteria and various parameters of the output images such as time complexity, quality, and clarity of the filtered image, ability to prevent original structure etc. The transition function may be single-level or multi-level. Also it may use concepts and features of some modern inventions like wavelet etc to further enhance the overall quality of the filtered images. The proposed filter may be used for better filtering and fast processing of video and other types of images used in medical science.

One of the advantages of the digital images is that it can be easily stored and processed in most of digital electronic gadgets like laptops and mobile phones. The proposed method is performing the filtering in the digital form of ultrasound images and it can be easily uploaded and processed in mobiles. The more efficient way is to design an app for the mobile and one can easily apply this algorithm through the mobile phone without the use of any expensive computer systems. 
Figure 5. Results of Various Filters for Image2 at noise density 0.01

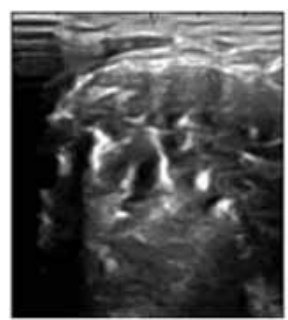

(a) Original

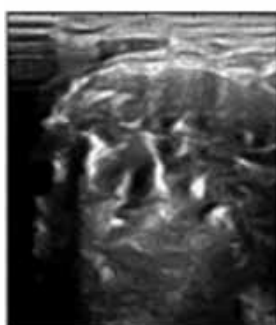

(f) Lee Diffusion

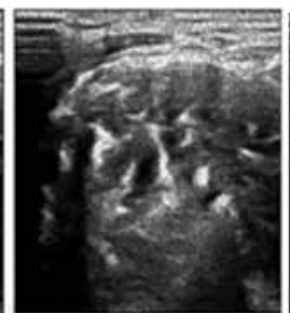

(b) Noise 0.01

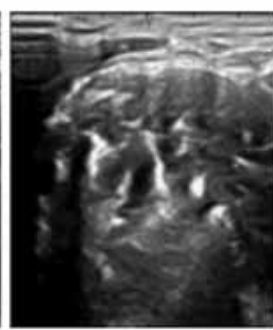

(g) Linear Scale

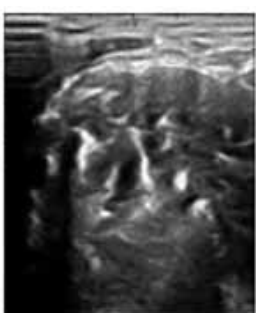

(c) Anistropic Diffusion



(h) SRAD

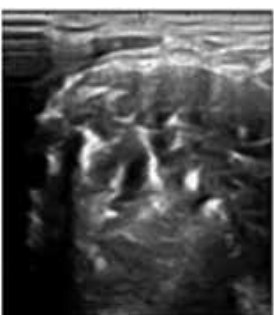

(d) Kuan



(i) Frost Diffusion

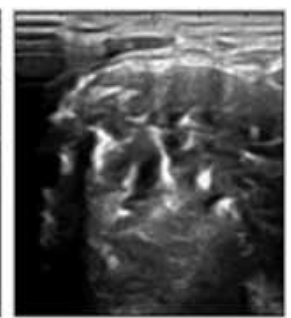

(e) Lee

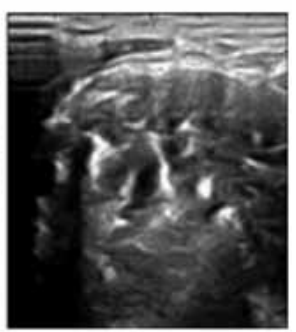

(j) Wavelet

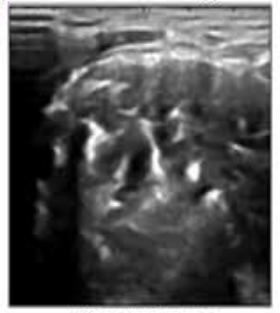

(k) CABDF 
Figure 6. Results of Various Filters for Image2 at Noise density 0.05

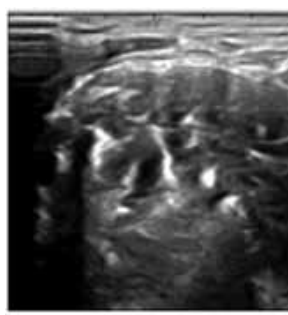

(a)Original

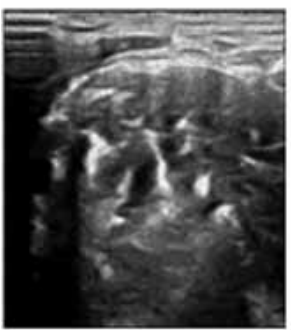

(f) Lee Diffusion

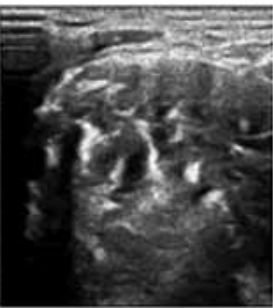

(b) Noise 0.05

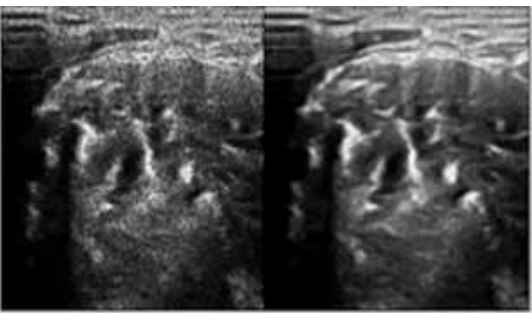

(c) Anistropic Diffusion

(d) Kuan

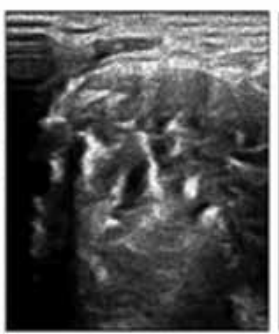

(h) SRAD

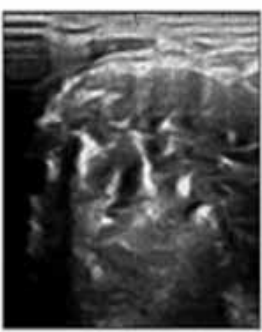

(i) Wavelet

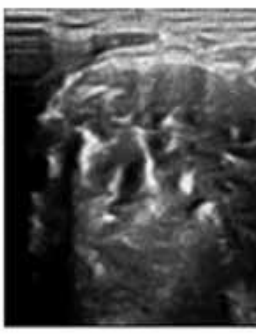

(g) Frost Diffusion

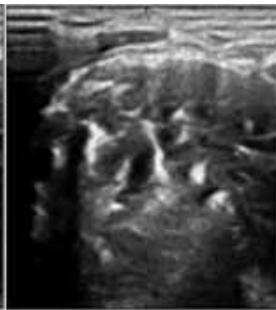

(e) Lee

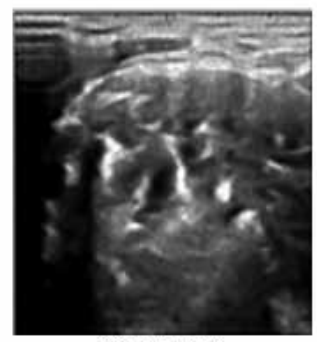

(k) CABDF

Figure 7. Comparison chart of various filters at noise 0.01 for image 1

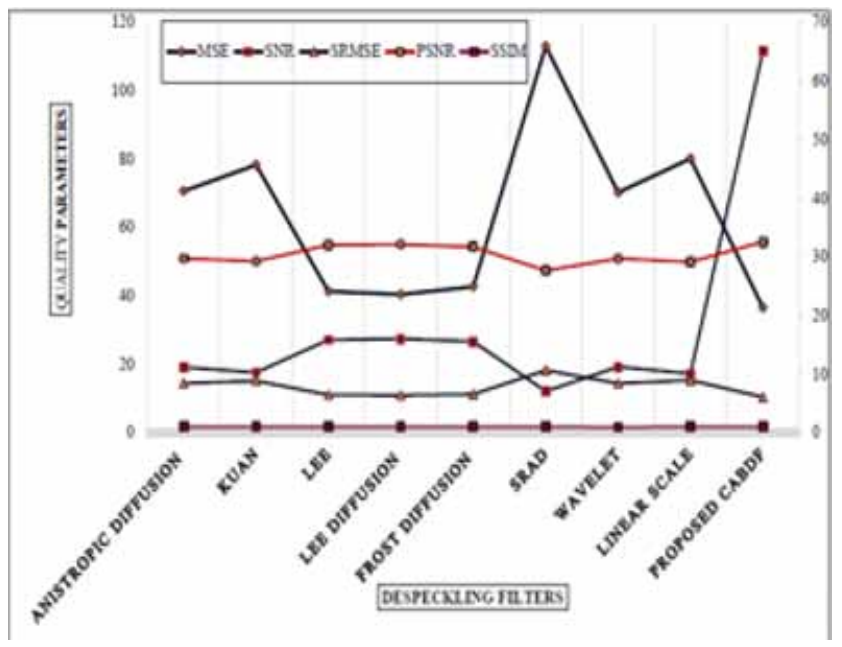


Figure 8. Comparison chart of various filters at noise 0.05 for image 1

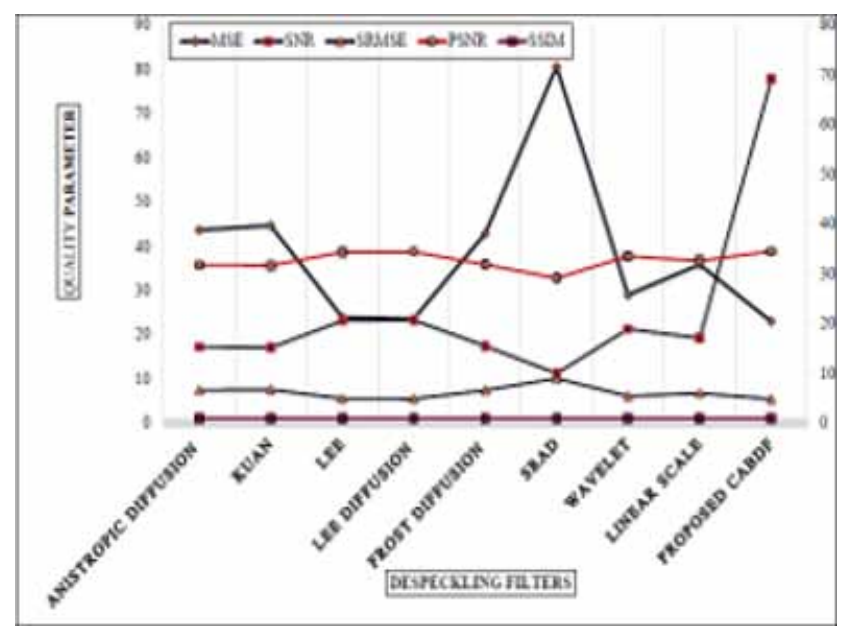

Figure 9. Comparison chart of various filters at noise 0.01 for image 2

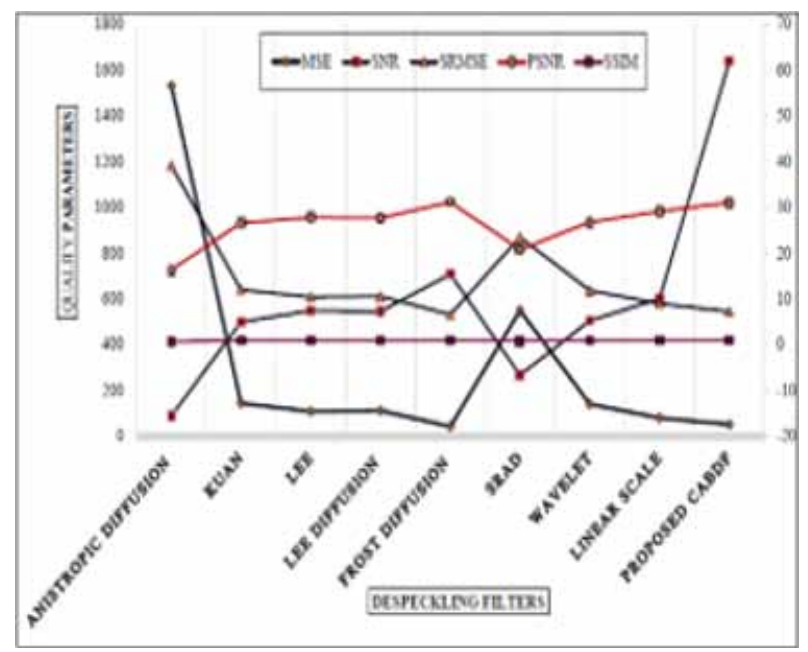


International Journal of E-Health and Medical Communications Volume $12 \cdot$ Issue $5 \cdot$ September-October 2021

Figure 10. Comparison chart of various filters at noise 0.05 for image 2

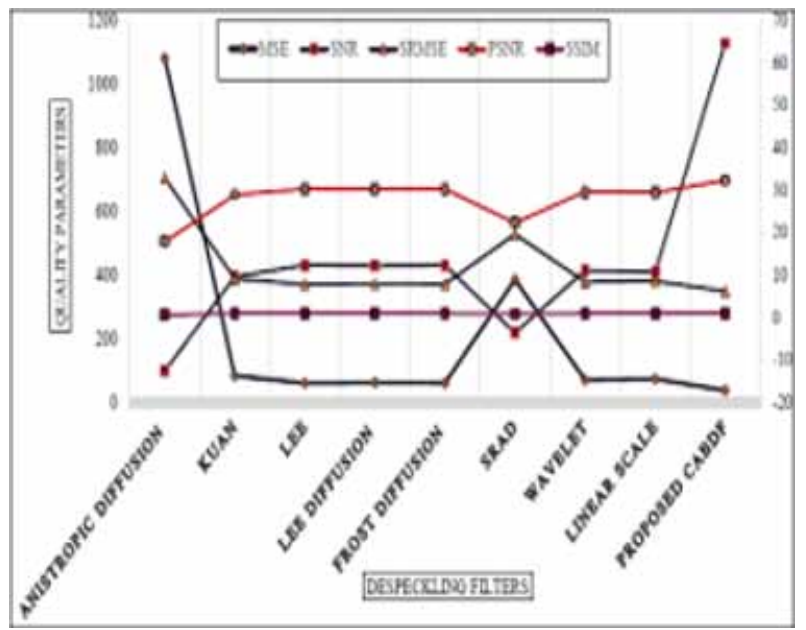




\section{REFERENCES}

Achim, A., Bezerianos, A., \& Tsakalides, P. (2001). Novel Bayesian multiscale method for speckle removal in medical ultrasound images. IEEE Transactions on Medical Imaging, 20(8), 772-783. doi:10.1109/42.938245 PMID: 11513028

Alex, D. M., \& Chandy, D. A. (2020, July). Evaluation of Inpainting in Speckled and Despeckled 2D Ultrasound Medical Images. In 2020 Advanced Computing and Communication Technologies for High Performance Applications (ACCTHPA) (pp. 221-225). IEEE.

Ambrosanio, M., Kanoun, B., \& Baselice, F. (2020). wKSR-NLM: An Ultrasound Despeckling Filter Based on Patch Ratio and Statistical Similarity. IEEE Access: Practical Innovations, Open Solutions, 8, 150773-150783. doi:10.1109/ACCESS.2020.3014909

Bhardwaj, A., Kaur, S., Shukla, A. P., \& Shukla, M. K. (2019, March). An Enhanced Cellular Automata Based Filter for Despeckling of Ultrasound Images. In 2019 6th International Conference on Signal Processing and Integrated Networks (SPIN) (pp. 1095-1099). IEEE. doi:10.1109/SPIN.2019.8711772

Bioucas-Dias, J. M., \& Figueiredo, M. A. (2010). Multiplicative noise removal using variable splitting and constrained optimization. IEEE Transactions on Image Processing, 19(7), 1720-1730. PMID:20215071

Chen, T. J., Chuang, K. S., Wu, J., Chen, S. C., Hwang, M., \& Jan, M. L. (2003). A novel image quality index using Moran I statistics. Physics in Medicine and Biology, 48(8), N131-N137. doi:10.1088/0031-9155/48/8/402 PMID:12741505

Cui, W., Li, M., Gong, G., Lu, K., Sun, S., \& Dong, F. (2020). Guided trilateral filter and its application to ultrasound image despeckling. Biomedical Signal Processing and Control, 55, 101625. doi:10.1016/j. bspc.2019.101625

Dabov, K., Foi, A., Katkovnik, V., \& Egiazarian, K. (2007). Image denoising by sparse 3-D transform-domain collaborative filtering. IEEE Transactions on Image Processing, 16(8), 2080-2095. doi:10.1109/TIP.2007.901238 PMID:17688213

Deledalle, C. A., Denis, L., Tabti, S., \& Tupin, F. (2017). MuLoG, or how to apply Gaussian denoisers to multichannel SAR speckle reduction? IEEE Transactions on Image Processing, 26(9), 4389-4403. doi:10.1109/ TIP.2017.2713946 PMID:28613174

Donoho, D. L., \& Johnstone, I. M. (1995). Adapting to unknown smoothness via wavelet shrinkage. Journal of the American Statistical Association, 90(432), 1200-1224. doi:10.1080/01621459.1995.10476626

Duarte-Salazar, C. A., Castro-Ospina, A. E., Becerra, M. A., \& Delgado-Trejos, E. (2020). Speckle Noise Reduction in Ultrasound Images for Improving the Metrological Evaluation of Biomedical Applications: An Overview. IEEE Access: Practical Innovations, Open Solutions, 8, 15983-15999. doi:10.1109/ACCESS.2020.2967178

Guo, Y., Cheng, H. D., Tian, J., \& Zhang, Y. (2009). A novel approach to speckle reduction in ultrasound imaging. Ultrasound in Medicine \& Biology, 35(4), 628-640. doi:10.1016/j.ultrasmedbio.2008.09.007 PMID:19243880

Guo, Y., Wang, Y., \& Hou, T. (2011). Speckle filtering of ultrasonic images using a modified non local-based algorithm. Biomedical Signal Processing and Control, 6(2), 129-138. doi:10.1016/j.bspc.2010.10.004

He, K., Sun, J., \& Tang, X. (2012). Guided image filtering. IEEE Transactions on Pattern Analysis and Machine Intelligence, 35(6), 1397-1409. doi:10.1109/TPAMI.2012.213 PMID:23599054

Hore, A., \& Ziou, D. (2010, August). Image quality metrics: PSNR vs. SSIM. In 2010 20th international conference on pattern recognition (pp. 2366-2369). IEEE https://www.kaggle.com/c/ultrasound-nerve-segmentation/data

Khare, A., Khare, M., Jeong, Y., Kim, H., \& Jeon, M. (2010). Despeckling of medical ultrasound images using Daubechies complex wavelet transform. Signal Processing, 90(2), 428-439. doi:10.1016/j.sigpro.2009.07.008

Knaus, C., \& Zwicker, M. (2013, September). Dual-domain image denoising. In 2013 IEEE International Conference on Image Processing (pp. 440-444). IEEE. doi:10.1109/ICIP.2013.6738091 
Kuan, D. T., Sawchuk, A. A., Strand, T. C., \& Chavel, P. (1985). Adaptive noise smoothing filter for images with signal-dependent noise. IEEE Transactions on Pattern Analysis and Machine Intelligence, PAMI-7(2), $165-177$. doi:10.1109/TPAMI.1985.4767641 PMID:21869255

Latif, G., Butt, M. O., Al Anezi, F. Y., \& Alghazo, J. (2020, October). Ultrasound Image Despeckling and detection of Breast Cancer using Deep CNN. In 2020 RIVF International Conference on Computing and Communication Technologies $(R I V F)$ (pp. 1-5). IEEE.

Lee, J. S. (1980). Digital image enhancement and noise filtering by use of local statistics. IEEE Transactions on Pattern Analysis and Machine Intelligence, PAMI-2(2), 165-168. doi:10.1109/TPAMI.1980.4766994 PMID:21868887

Lee, J. S., Wen, J. H., Ainsworth, T. L., Chen, K. S., \& Chen, A. J. (2008). Improved sigma filter for speckle filtering of SAR imagery. IEEE Transactions on Geoscience and Remote Sensing, 47(1), 202-213.

Loizou, C. P., Theofanous, C., Pantziaris, M., \& Kasparis, T. (2014). Despeckle filtering software toolbox for ultrasound imaging of the common carotid artery. Computer Methods and Programs in Biomedicine, 114(1), 109-124. doi:10.1016/j.cmpb.2014.01.018 PMID:24560276

Mangalam, K., \& Venkatesh, K. S. (2017, June). Bitwise operations of cellular automaton on gray-scale images. In 2017 28th Irish Signals and Systems Conference (ISSC) (pp. 1-5). IEEE. doi:10.1109/ISSC.2017.7983625

Mei, K., Hu, B., Fei, B., \& Qin, B. (2019). Phase Asymmetry Ultrasound Despeckling With Fractional Anisotropic Diffusion and Total Variation. IEEE Transactions on Image Processing, 29, 2845-2859. doi:10.1109/ TIP.2019.2953361 PMID:31751240

Nowak, R. D. (1999). Wavelet-based Rician noise removal for magnetic resonance imaging. IEEE Transactions on Image Processing, 8(10), 1408-1419. doi:10.1109/83.791966 PMID:18267412

Paul, A., Mukherjee, D. P., \& Acton, S. T. (2019, September). Shape Based Speckle Removal for Ultrasound Image Segmentation. In 2019 IEEE International Conference on Image Processing (ICIP) (pp. 3586-3590). IEEE. doi:10.1109/ICIP.2019.8803493

Perona, P., \& Malik, J. (1990). Scale-space and edge detection using anisotropic diffusion. IEEE Transactions on Pattern Analysis and Machine Intelligence, 12(7), 629-639. doi:10.1109/34.56205

Popovici, A., \& Popovici, D. (2002, August). Cellular automata in image processing. In Fifteenth International Symposium on Mathematical Theory of Networks and Systems (Vol. 1, pp. 1-6). Academic Press.

Prakash, K. B., Babu, R. V., \& Gopal, B. V. (2011). Image independent filter for removal of speckle noise. International Journal of Computer Science Issues, 8(5), 196.

Ramos-Llordén, G., Vegas-Sánchez-Ferrero, G., Martin-Fernandez, M., Alberola-López, C., \& Aja-Fernández, S. (2014). Anisotropic diffusion filter with memory based on speckle statistics for ultrasound images. IEEE Transactions on Image Processing, 24(1), 345-358. doi:10.1109/TIP.2014.2371244 PMID:25415987

Rotman, S. N., Friedman, Z., \& Porat, M. (2019, October). Simultaneous Compression and De-Speckling of Medical Ultrasound Images. In 2019 IEEE International Ultrasonics Symposium (IUS) (pp. 1448-1450). IEEE. doi:10.1109/ULTSYM.2019.8925852

Rukmini, V., \& Singh, M. D. (2008). Filter selection for speckle noise reduction (Doctoral dissertation).

Sasikala, D., \& Madheswaran, M. (2014). Speckle noise filtering for ultrasound images of common carotid artery: a review. Ictact Journal on Image \& Video Processing, 4(4).

Selvapeter, P. J., \& Hordijk, W. (2009, December). Cellular automata for image noise filtering. In 2009 World Congress on Nature \& Biologically Inspired Computing (NaBIC) (pp. 193-197). IEEE. doi:10.1109/ NABIC.2009.5393684

Shukla, A. P., \& Agarwal, S. (2014, November). Training cellular automata for salt and pepper noise filtering. In 2014 Innovative Applications of Computational Intelligence on Power, Energy and Controls with their impact on Humanity (CIPECH) (pp. 519-524). IEEE.

Singh, L., Singh, A. K., \& Singh, P. K. (2020). Secure data hiding techniques: A survey. Multimedia Tools and Applications, 79(23), 15901-15921. doi:10.1007/s11042-018-6407-5 
Tiwari, A., Singh, P. K., \& Amin, S. (2016, January). A survey on shadow detection and removal in images and video sequences. In 2016 6th International Conference-Cloud System and Big Data Engineering (Confluence) (pp. 518-523). IEEE.

Touzi, R. (2002). A review of speckle filtering in the context of estimation theory. IEEE Transactions on Geoscience and Remote Sensing, 40(11), 2392-2404. doi:10.1109/TGRS.2002.803727

Ulam, S. M., \& SM, U. (1972). Some ideas and prospects in biomathematics. Academic Press.

Wolfram, S. (1984). Computation theory of cellular automata. Communications in Mathematical Physics, 96(1), 15-57. doi:10.1007/BF01217347

Yu, Y., \& Acton, S. T. (2002). Speckle reducing anisotropic diffusion. IEEE Transactions on Image Processing, 11(11), 1260-1270. doi:10.1109/TIP.2002.804276 PMID:18249696

Zhang, J., Lin, G., Wu, L., \& Cheng, Y. (2016). Speckle filtering of medical ultrasonic images using wavelet and guided filter. Ultrasonics, 65, 177-193. doi:10.1016/j.ultras.2015.10.005 PMID:26489484

Zhou, S. K., Rueckert, D., \& Fichtinger, G. (Eds.). (2019). Handbook of medical image computing and computer assisted intervention. Academic Press.

Ankur Bhardwaj received an M.Tech degree in Computer Science and Engineering from Teerthanker Mahavir University Moradabad in 2012. He is pursuing his PhD from Amity School of Engineering and Technology, Amity University, Noida. He has 14 years of teaching experience teaching undergraduate and postgraduate students. He has two patents and authored more than 10 papers in international Scopus indexed conferences and journals. His area of interest is medical image processing.

Sanmukh Kaur received an M.Tech degree (with distinction) in Electronics and Communication Engineering from PEC University of Technology Chandigarh in 2001. She did her PhD from Thapar University Patiala in July 2015. She has 17 years of teaching experience teaching undergraduate and postgraduate students. She has filed eight patents, authored more than twenty papers in international SCl and Scopus indexed journals and authored a number of papers in national and international conferences and journals. Her area of interest is optical fiber and wireless communication, optical devices, and free space optics.

Anand Prakash Shukla is working as Professor in CSE Dept. at KIET Group of Institutions Ghaziabad. He received B.E. (Computer Science \& Engineering), M.Tech. (Computer Science), and Ph.D. Degree in Computer Science and Engineering from MNNIT Allahabad. He has 20 years of experience in teaching and research in computer science and engineering. He has several international and Indian patents, research papers, books and articles.

Manoj Kumar Shukla is working as Associate Professor in Computer Science \& Engineering department at Amity University Noida. He received an M. Tech degree from GGIP University, Delhi in 2010. He received dual PhD degree from California University, USA in 2012 and from Indian Institute of Mines, Dhanbad in 2015. He has 11 years of teaching experience in teaching and research. He authored more than forty five research papers in national and international conferences and journals. He has several international and Indian patents and articles. 